\title{
REYNOLDS STRESS MODEL ADJUSTMENTS FOR SEPARATED FLOWS
}

\author{
ALEXEY TROSHIN ${ }^{1,2}$, IVAN MATYASH' ${ }^{1}$ AND SERGEY MIKHAYLOV ${ }^{1,2}$ \\ ${ }^{1}$ Central Aerohydrodynamic Institute \\ 1 Zhukovsky str., Zhukovsky, Moscow region, 140180 Russia \\ ai-troshin@yandex.ru \\ ${ }^{2}$ Moscow Institute of Physics and Technology \\ 9 Institutskiy per., Dolgoprudny, Moscow region, 141701 Russia
}

Key words: Turbulence model, Reynolds stress, Separation.

\begin{abstract}
The development of a new Reynolds stress model suitable for computations of separated flows is described. It is based on the recently published version of SSG/LRR- $\omega$ model, which includes a correction eliminating streamline back-bending near the reattachment point. Modifications are proposed which reduce the turbulence kinetic energy dissipation rate and pressure-strain term near the separation point. The validation results using the Diverging channel flow test case are presented. The quality of the obtained results is assessed.
\end{abstract}

\section{INTRODUCTION}

The problem of unreliable prediction of separation zones in the framework of the Reynolds approach is widely known. Both linear eddy viscosity models [1] and differential Reynolds stress models [2] can significantly impair the characteristics of separation zones. An analysis of the flow fields shows that the main feature that turbulence models do not describe is high level of velocity fluctuations in the vicinity of the separation point in the mixing layer [3].

The mixing layer which bounds the separation zone undergoes "secondary turbulization" and passes through the period of existence of ordered quasi-two-dimensional large eddies [4]. These vortices exist for some time, after which they collapse, turning into a set of smaller randomly oriented vortex structures, and generate quasi-equilibrium turbulence. Throughout this process, the production of kinetic energy of turbulence

$$
P=-R_{i j} \frac{\partial \tilde{u}_{i}}{\partial x_{j}} \approx-R_{x y} \frac{\partial \tilde{u}}{\partial y}, \text { where } R_{i j}=u_{i} u_{j},
$$

has the same order of magnitude since it is associated with the interaction of large-scale structures with mean velocity field. In contrast, the turbulence kinetic energy dissipation rate

$$
\varepsilon=-\overline{\tau_{i j}^{\prime} \frac{\partial u_{i}^{\prime}}{\partial x_{j}}}, \text { where } \tau_{i j}=-\mu\left(\frac{\partial u_{i}}{\partial x_{j}}+\frac{\partial u_{j}}{\partial x_{i}}-\frac{2}{3} \frac{\partial u_{m}}{\partial x_{m}} \delta_{i j}\right),
$$


is small at the beginning of the process. While small-scale turbulence has not yet formed, the flow state with $\varepsilon<<P$ is observed which generates high levels of fluctuations in the vicinity of the separation point. Only later, after the destruction of ordered vortices, the turbulent energy cascade reaches a quasi-equilibrium state with $P \sim \varepsilon$. Similar reasoning allows us to expect the action of the pressure-strain term to be also suppressed in the nonequilibrium turbulence region of such type.

Present-day turbulence models are focused on quasi-equilibrium flows with $P \sim \varepsilon$ (boundary layers, mixing layers, jets, wakes), and do not correctly predict the first stage of the mixing layer development at the separation zone boundary, where $\varepsilon<<P$. There are known attempts to eliminate this defect within the framework of the stationary RANS approach [3]. In this paper, we propose another way to solve this problem which does not involve the second derivatives of the velocity field.

\section{STARTING POINT FOR BUILDING A NEW MODEL}

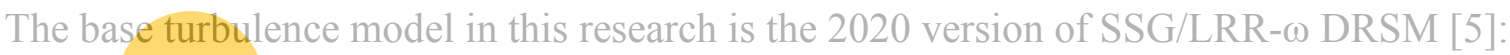
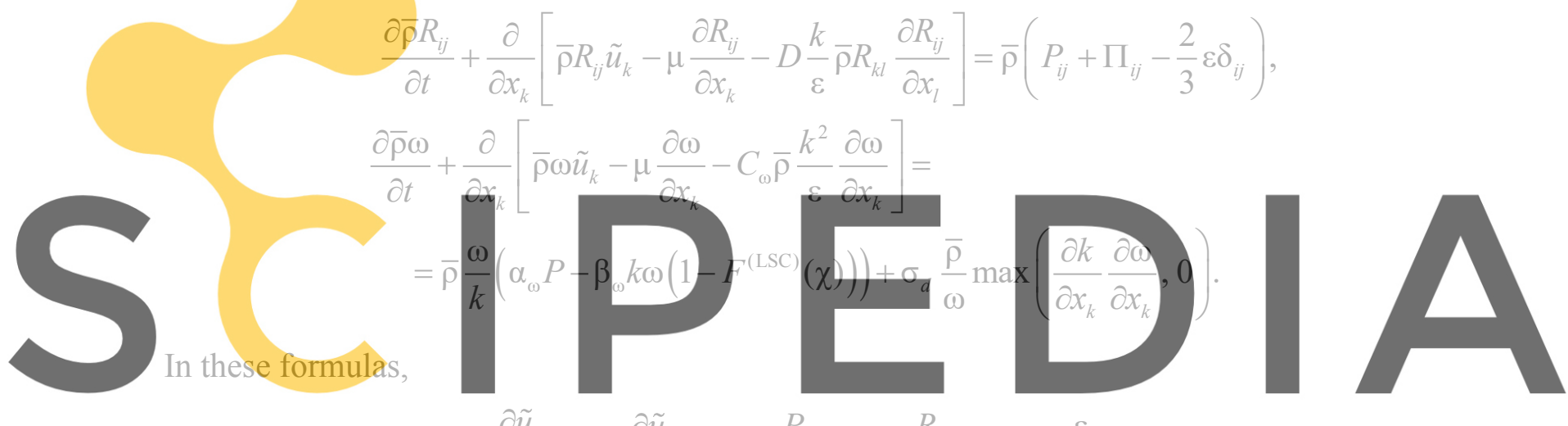

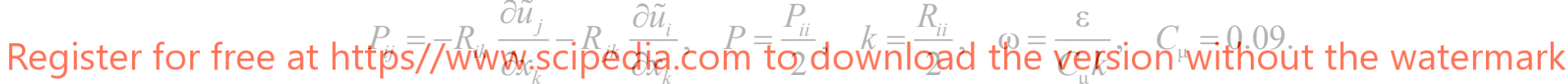

Pressure-strain term model is given by the formula

$$
\begin{aligned}
\Pi_{i j}=-\left(C_{1} \varepsilon+C_{1}^{*} P\right) a_{i j} & +C_{2} \varepsilon\left(a_{i k} a_{k j}-\frac{1}{3} A_{2} \delta_{i j}\right)+\left(C_{3}-C_{3}^{*} \sqrt{a_{k l} a_{k l}}\right) k S_{i j}^{*}+ \\
& +C_{4} k\left(a_{i k} S_{k j}+S_{i k} a_{k j}-\frac{2}{3} a_{k l} S_{k l} \delta_{i j}\right)+C_{5} k\left(\Omega_{i k} a_{k j}-a_{i k} \Omega_{k j}\right) .
\end{aligned}
$$

The following notation is introduced:

$$
a_{i j}=\frac{R_{i j}}{k}-\frac{2}{3} \delta_{i j}, \quad \Omega_{i j}=\frac{1}{2}\left(\frac{\partial \tilde{u}_{i}}{\partial x_{j}}-\frac{\partial \tilde{u}_{j}}{\partial x_{i}}\right), \quad S_{i j}=\frac{1}{2}\left(\frac{\partial \tilde{u}_{i}}{\partial x_{j}}+\frac{\partial \tilde{u}_{j}}{\partial x_{i}}\right), \quad S_{i j}^{*}=\tilde{S}_{i j}-\frac{1}{3} \frac{\partial \tilde{u}_{m}}{\partial x_{m}} \delta_{i j} .
$$

Additional term $F^{(\mathrm{LSC})}$ (LSC stands for "length-scale correction") depends in the nearest wall distance $d_{\text {wall }}$. It is responsible for correct shape of streamlines in the vicinity of reattachment point: 


$$
\begin{gathered}
F^{(\mathrm{LSC})}(\chi)=\frac{1+\operatorname{th}\left(A \cdot\left(\chi-\chi_{T}\right)\right)}{2}, \quad \chi=\max \left[\left(\frac{L_{t}}{L_{\log }}-1\right)\left(\frac{L_{t}}{L_{\log }}\right)^{2}, 0\right], \\
\frac{L_{t}}{L_{\log }}=\frac{\sqrt{k}}{C_{\mu}^{1 / 4} \kappa \omega d_{\text {wall }}}, \quad \kappa=0.41, \quad A=31, \quad \chi_{T}=1.0 .
\end{gathered}
$$

The SSG/LRR- $\omega$ model contains two sets of coefficients: near-wall (first) and freeturbulent (second), with blending function $F_{1}$ to switch between them using the formula $C=C_{1} F_{1}+C_{2}\left(1-F_{1}\right)$.

This blending function is taken from SST model [6]:

$$
F_{1}=\operatorname{th}\left(\arg _{1}^{4}\right), \arg _{1}=\min \left(\max \left(\frac{\sqrt{k}}{C_{\mu} \omega d_{\text {wall }}}, \frac{500 v}{\omega d_{\text {wall }}^{2}}\right), \frac{4 \rho \sigma_{\omega 2} k}{\mathrm{CD} \cdot d_{\text {wall }}^{2}}\right), \mathrm{CD}=\frac{\sigma_{d 2}}{\omega} \max \left(\frac{\partial k}{\partial x_{k}} \frac{\partial \omega}{\partial x_{k}}, 0\right) .
$$

\section{Coefficient values of SSG/LRR- $\omega$ model are collected in Table 1.}

Table 1: Coefficient values of SSG/LRR- $\omega$ model

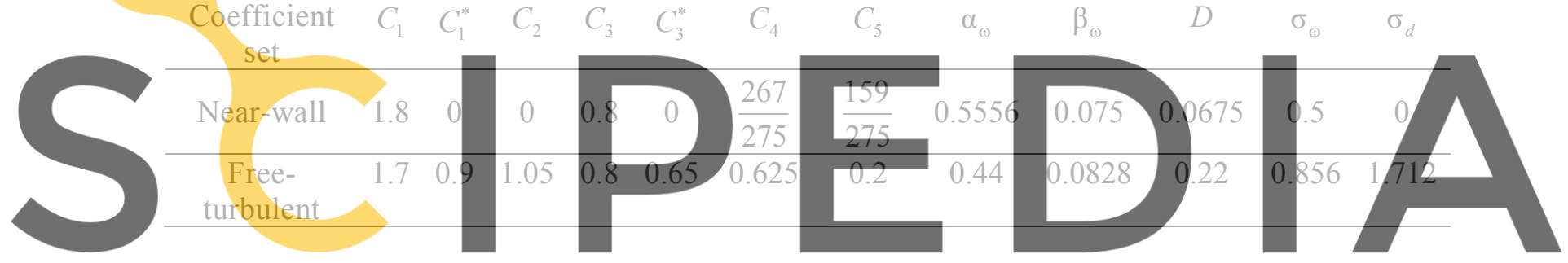

Let us consider the Periodic hill flow at Reynolds number $R e=37000$ [7]. The

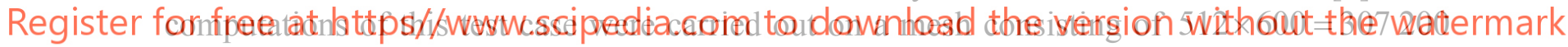

cells selected on a basis of mesh convergence analysis. TsAGI in-house code zFlare was used with $2^{\text {nd }}$ order TVD scheme. The solution obtained with base model is shown in Fig. 1. Reattachment point coordinate was found to be $x_{r} / h=5.58$, which is $48 \%$ farther from the hill crest than experimental value $x_{r} / h=3.76$.

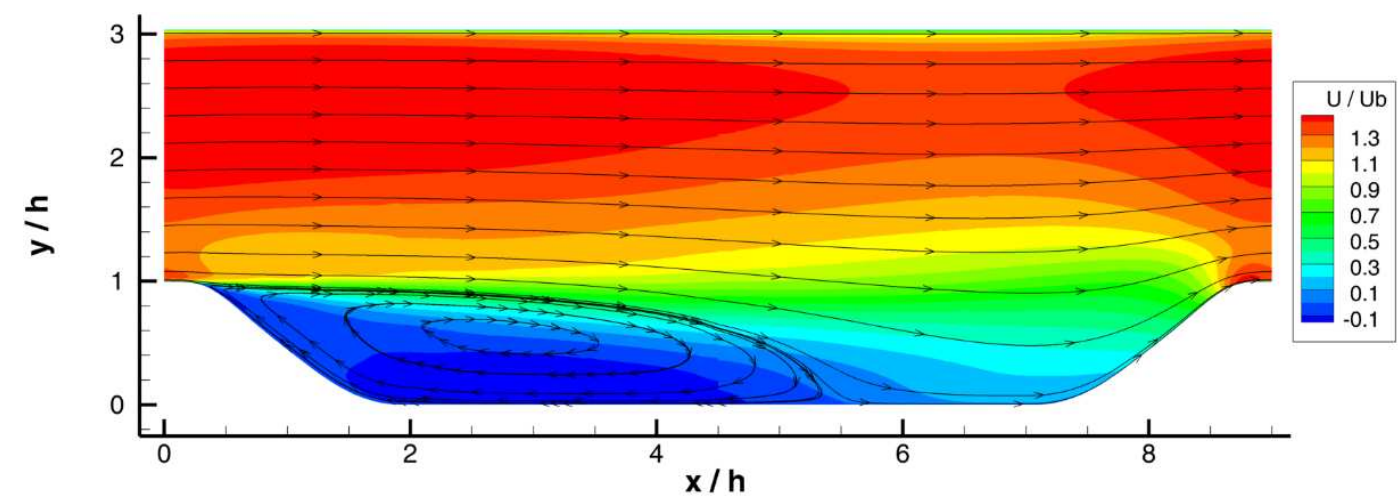

Figure 1: Mean velocity field and streamlines in the computation using base SSG/LRR- $\omega$ model 


\section{DEVELOPMENT OF SEPARATION INDICATOR FUNCTION}

We propose to start from a geometric view of the separation region. We will identify it by the angle between the streamlines and the wall plane. If there is no separation, the flow is almost parallel to the wall, so the angle is small. At the separation origin, the flow moves away from the wall, and the angle becomes significant.

The streamlines are determined by the mean velocity field $\mathbf{V}(x, y, z)$, but the direct use of $\mathbf{V}$ in the indicator is undesirable: this leads to the Galilean non-invariance of the model. One should find a parameter that does not depend on the velocity field itself, but only on its gradients. Consider the expression

$$
N_{1}=\frac{1}{\sqrt{C_{\mu}} \omega} \frac{\partial \tilde{u}_{i}}{\partial x_{j}} n_{i} n_{j}
$$

where $\mathrm{n}=\nabla d_{\text {wall }} /\left|\nabla d_{\text {wall }}\right|$ is the wall-normal unit vector. By design, $N_{1}$ is tensorially and Galilean invariant. Imagine a wall-parallel flow with normal vector $\mathbf{n}=(0,1,0)$. In this case,

$$
N_{1}=\frac{1}{\sqrt{C_{\mu}} \omega} \frac{\partial \tilde{v}}{\partial y} \approx 0
$$

If the flow, again directed along the $x$-axis, is perpendicular to the wall, as, for example, in the case of a mixing layen
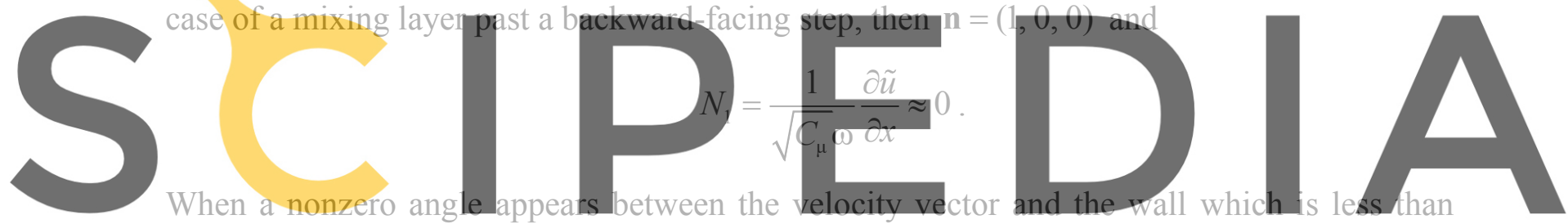

$\pi / 2, N_{1}$ value becomes different from 0 . For example, if $\boldsymbol{n}=(1 / \sqrt{2}, 1 / \sqrt{2}, 0)$, we will get

Register for free at https//www.scipedia.com to download the version without the watermark

$$
N_{1}=\frac{1}{\sqrt{C_{\mu}} \omega}\left(\frac{1}{2} \frac{\partial \tilde{u}}{\partial x}+\frac{1}{2} \frac{\partial \tilde{u}}{\partial y}+\frac{1}{2} \frac{\partial \tilde{v}}{\partial x}+\frac{1}{2} \frac{\partial \tilde{v}}{\partial y}\right) \approx \frac{1}{2 \sqrt{C_{\mu}} \omega} \frac{\partial \tilde{u}}{\partial y}
$$

It is seen that $N_{1}$ is proportional to main velocity gradient $\partial \tilde{u} / \partial y$.

Consider $N_{1}$ field computed using SSG/LRR- $\omega$ solution of Periodic hill flow, see Fig. 2. This parameter clearly identifies the separation zone $\left(N_{1}>0\right)$ and the region where flow approaches the hill crest in the right part of the computational domain $\left(N_{1}<0\right)$. In addition, spurious region is identified above the separation $(0<x / h<2,1.5<y / h<2)$.

Note (see Fig. 3) that mixing layer region is reliably identified with the third invariant of the stress anisotropy tensor, $A_{3}=a_{i j} a_{j k} a_{k i}$. Using this fact, let us multiply $N_{1}$ by $12 A_{3}$ :

$$
N_{2}=12 A_{3} N_{1}=\frac{12 A_{3}}{\sqrt{C_{\mu}} \omega} \frac{\partial \tilde{u}_{i}}{\partial x_{j}} n_{i} n_{j} .
$$




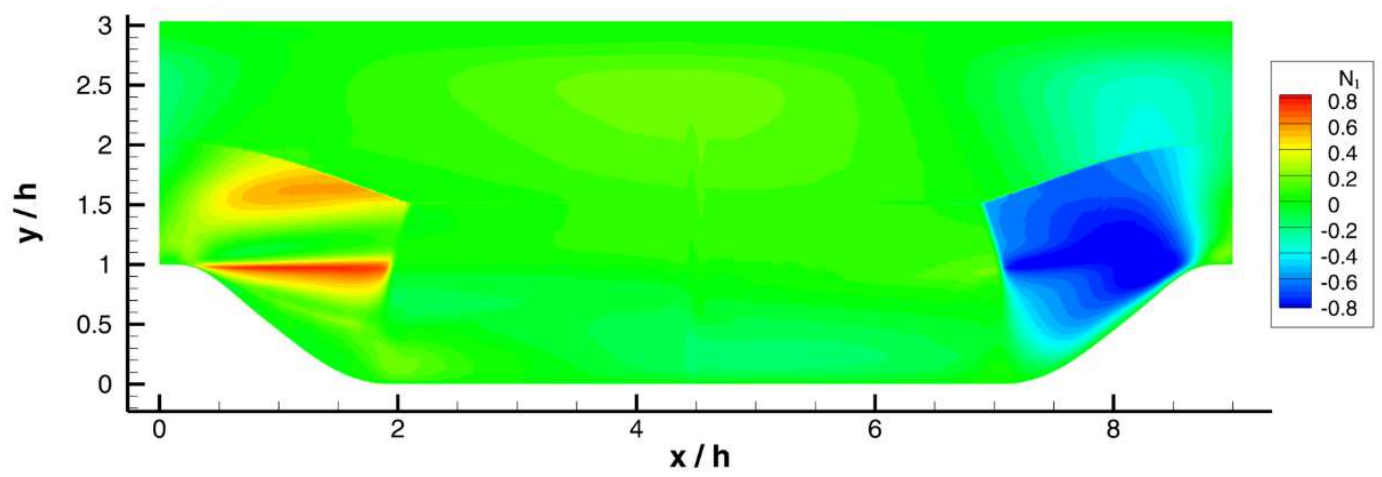

Figure 2: $N_{1}$ parameter field

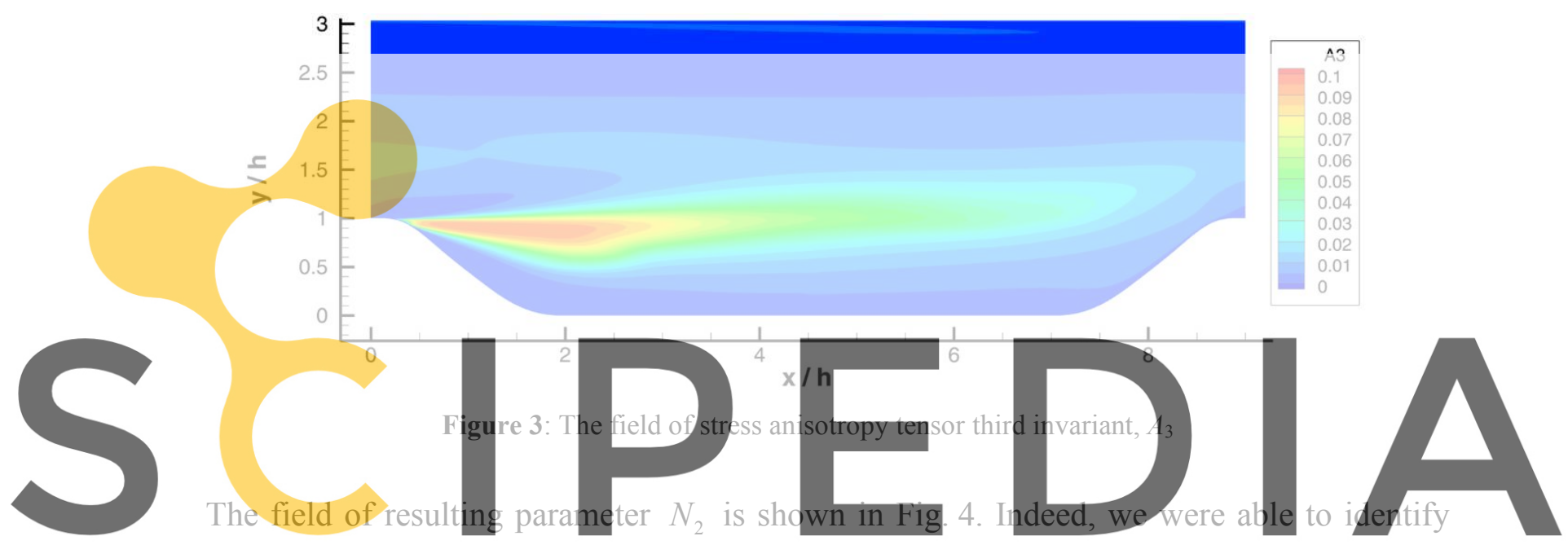

the separation origin region virtually without spurious zones. It remains to ensure that the

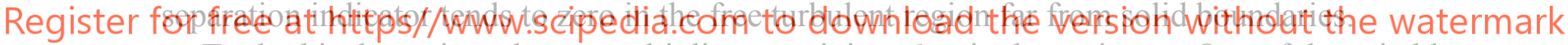

To do this, let us introduce a multiplier containing $d_{\text {wall }}$ in denominator. One of the suitable

expressions may be a formula based on (1): $I_{\text {wall }}=\min \left\{C_{\text {wall }} \sqrt{k} /\left(\omega d_{\text {wall }}\right), 1\right\}$. Coefficient $C_{\text {wall }}$ equal to 30 and $I_{\text {wall }}$ limiting from above to 1 are introduced since in addition to freeturbulent zones, $C_{\text {wall }} \sqrt{k} /\left(\omega d_{\text {wall }}\right)$ decreases noticeably in separation mixing layer region. This behavior should be compensated for. As a result, the following expression for the indicator function is proposed: $I_{\text {sep }}=\min \left\{\max \left\{C_{\text {sep }} N_{2} I_{\text {wall }}, 0\right\}, 1\right\}$. The indicator is again limited to remain inside $[0,1]$ interval under any conditions. Coefficient $C_{\text {sep }}$ is introduced for calibration. $I_{\text {sep }}$ field with $C_{\text {sep }}=1$ is shown in Fig. 5. 


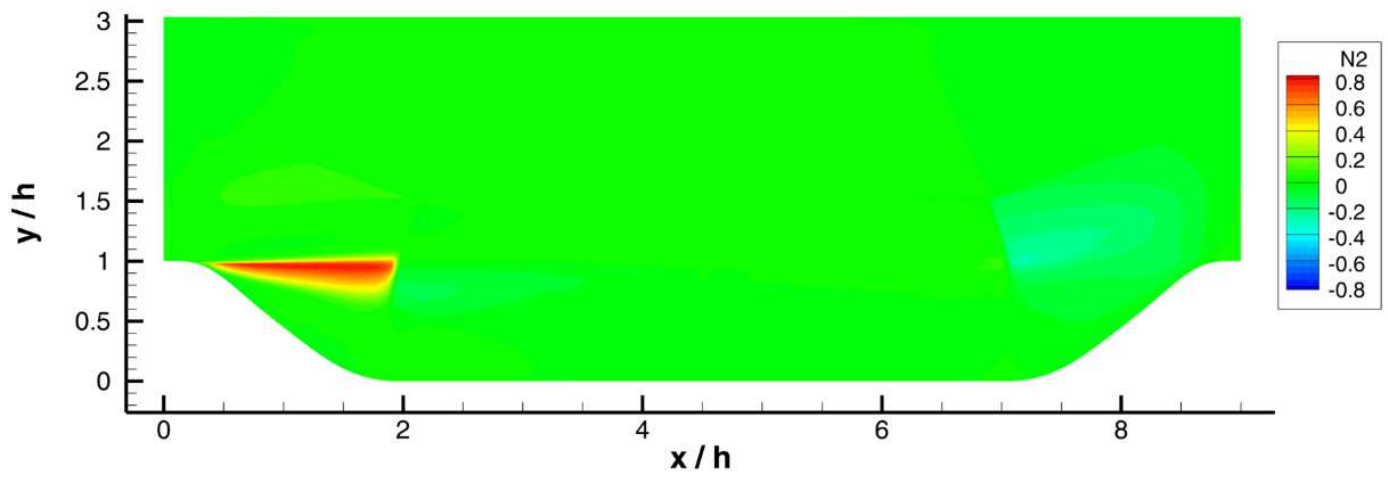

Figure 4: $N_{2}$ parameter field
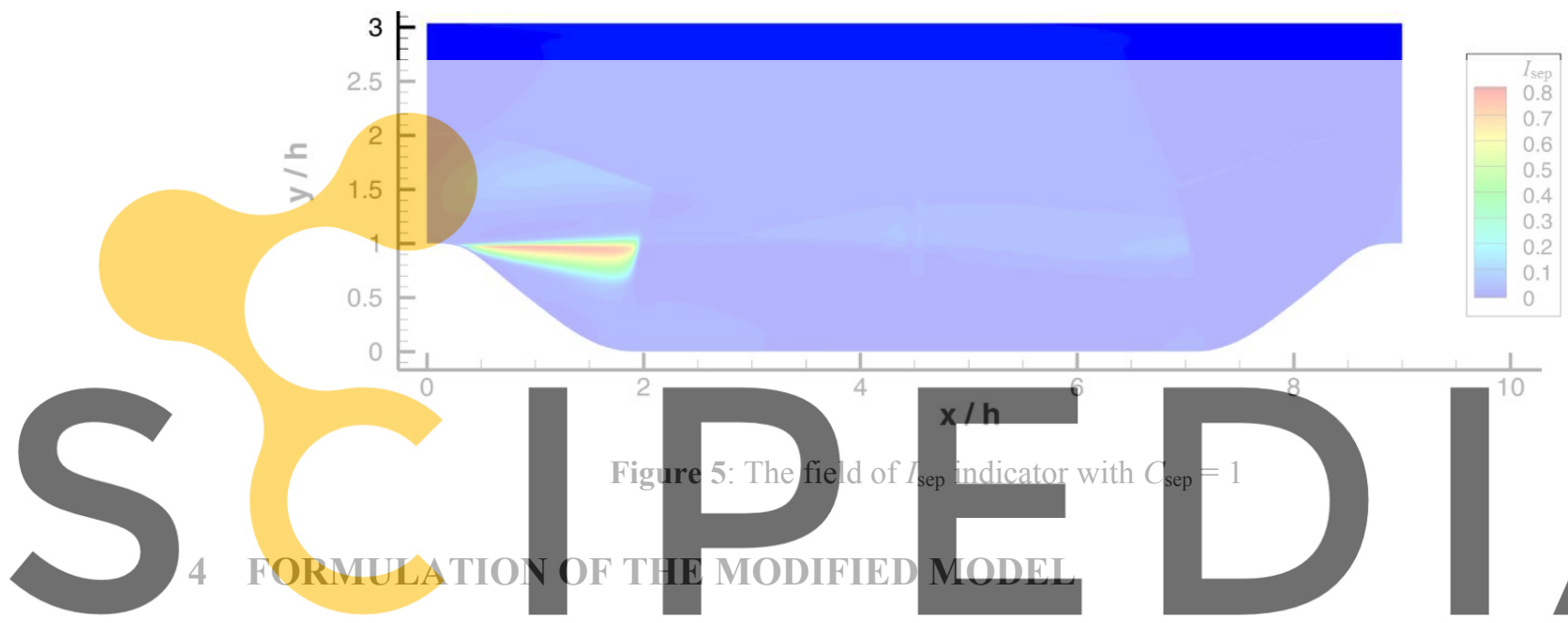

To reduce the dissipation rate at the separation zone origin, we multiply $\varepsilon$ in the $R_{i j}$

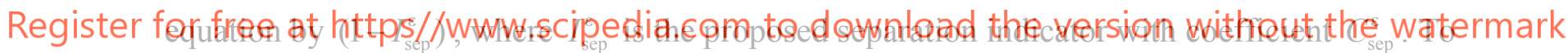

study the effect of $C_{\text {sep }}^{\varepsilon}$ value, computations were carried out with $C_{\text {sep }}^{\varepsilon}=\{1.0,3.0,5.0\}$. Fig. 6

shows the response of the turbulence kinetic energy field to $C_{\text {sep }}^{\varepsilon}$ variation.

Qualitative transformation of $k$ field is seen. Now, instead of a slow increase in energy along the mixing layer until reaching the equilibrium value at $x / h=4$, a sharp energy peak at $x / h \approx 1$ is observed. This behavior is better consistent with the experimental data than the behavior of the original model.

We will try to achieve further improvements by modifying the pressure-strain term model. Let us introduce the separation indicators to $\Pi_{i j}$ as follows:

$$
\begin{aligned}
\Pi_{i j} & =\left[-C_{1} a_{i j}+C_{2}\left(a_{i k} a_{k j}-\frac{1}{3} A_{2} \delta_{i j}\right)\right] \cdot \varepsilon \cdot\left(1-I_{\mathrm{sep}}^{\varepsilon}\right)-C_{1}^{*} P a_{i j}+ \\
& +\left(C_{3}-C_{3}^{*} \sqrt{A_{2}}\right) k S_{i j}^{*} \cdot\left(1-I_{\mathrm{sep}}^{\phi}\right)+C_{4} k\left(a_{i k} S_{k j}+S_{i k} a_{k j}-\frac{2}{3} a_{k l} S_{k l} \delta_{i j}\right)+C_{5} k\left(\Omega_{i k} a_{k j}-a_{i k} \Omega_{k j}\right) .
\end{aligned}
$$



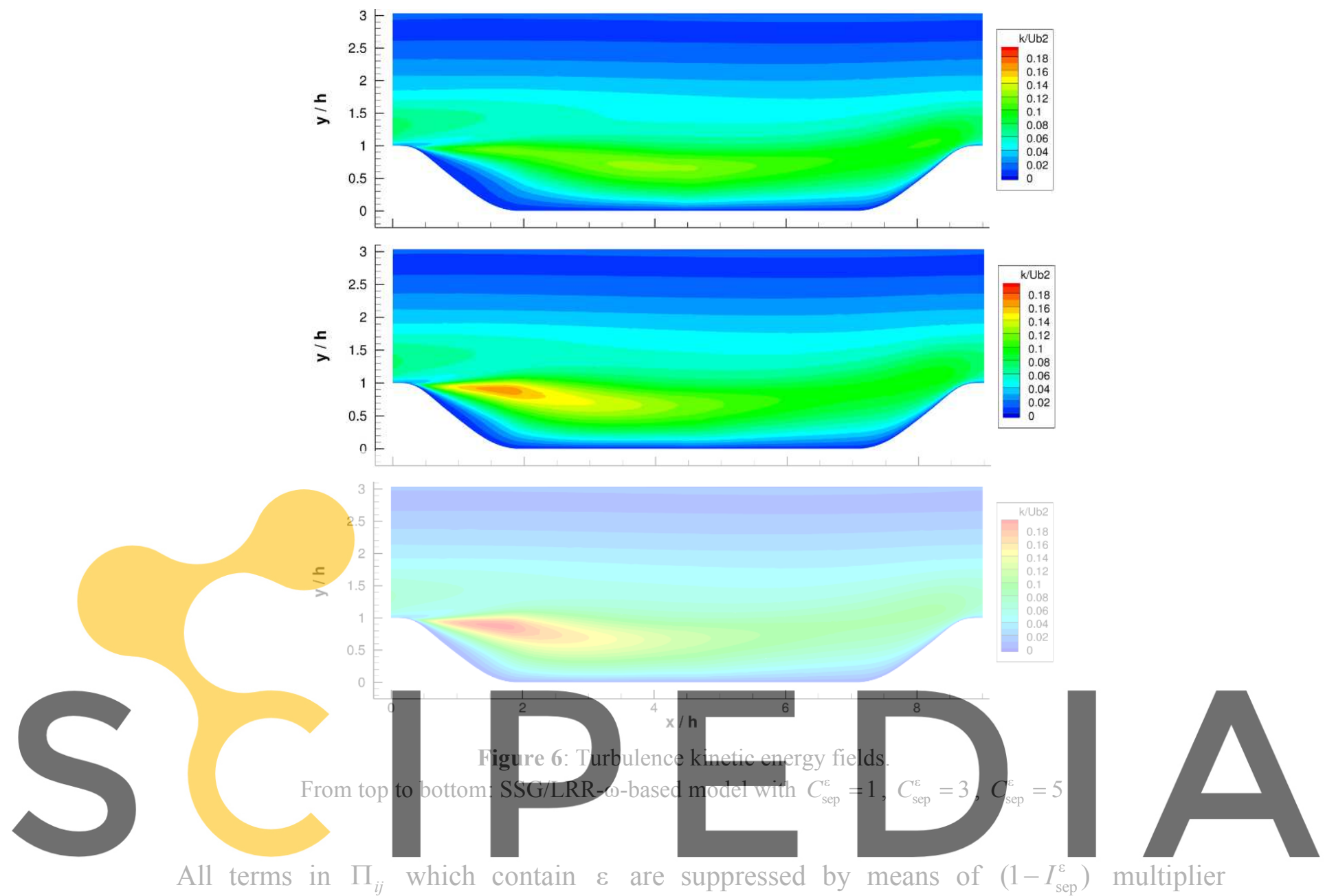

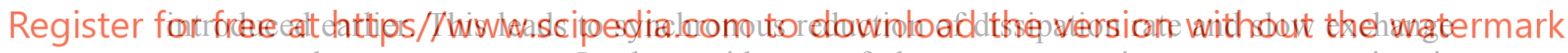
processes between stresses. In the rapid part of the pressure-strain term, suppression is introduced only in the term proportional to $k S_{i j}^{*}$. It has zeroth order in the anisotropy tensor and should give the greatest effect when modified. The $I_{\text {sep }}^{\phi}$ term is different from $I_{\text {sep }}^{\varepsilon}$ only in coefficient, which is now $C_{\text {sep }}^{\phi}$. Thus, we have two degrees of freedom to adjust the separation zone length and stress level.

The algorithm for joint calibration of coefficients $C_{\text {sep }}^{\varepsilon}$ and $C_{\text {sep }}^{\phi}$ is as follows:

1. By varying $C_{\text {sep }}^{\varepsilon}$, the value of $\max _{x / h=1} R_{x x}$ equal to $0.074 \pm 0.07$ is achieved.

2. By varying $C_{\text {sep }}^{\phi}$, the reattachment point coordinate $x_{r} / h=3.76 \pm 0.3$ is achieved.

3. If after step 2 the $\max _{x / h=1} R_{x x}$ has gone beyond the desired range, return to step 1 .

The calibration process converged to the values $C_{\text {sep }}^{\varepsilon}=0.85, C_{\text {sep }}^{\phi}=1.32$ in 3 iterations. The solution obtained with these coefficients is compared with the basic SSG/LRR- $\omega$ model and experimental data in Fig. 7. The separation zone size was obtained which differs from the experiment by no more than $2 \%$, while basic model overestimated it by $48 \%$. 

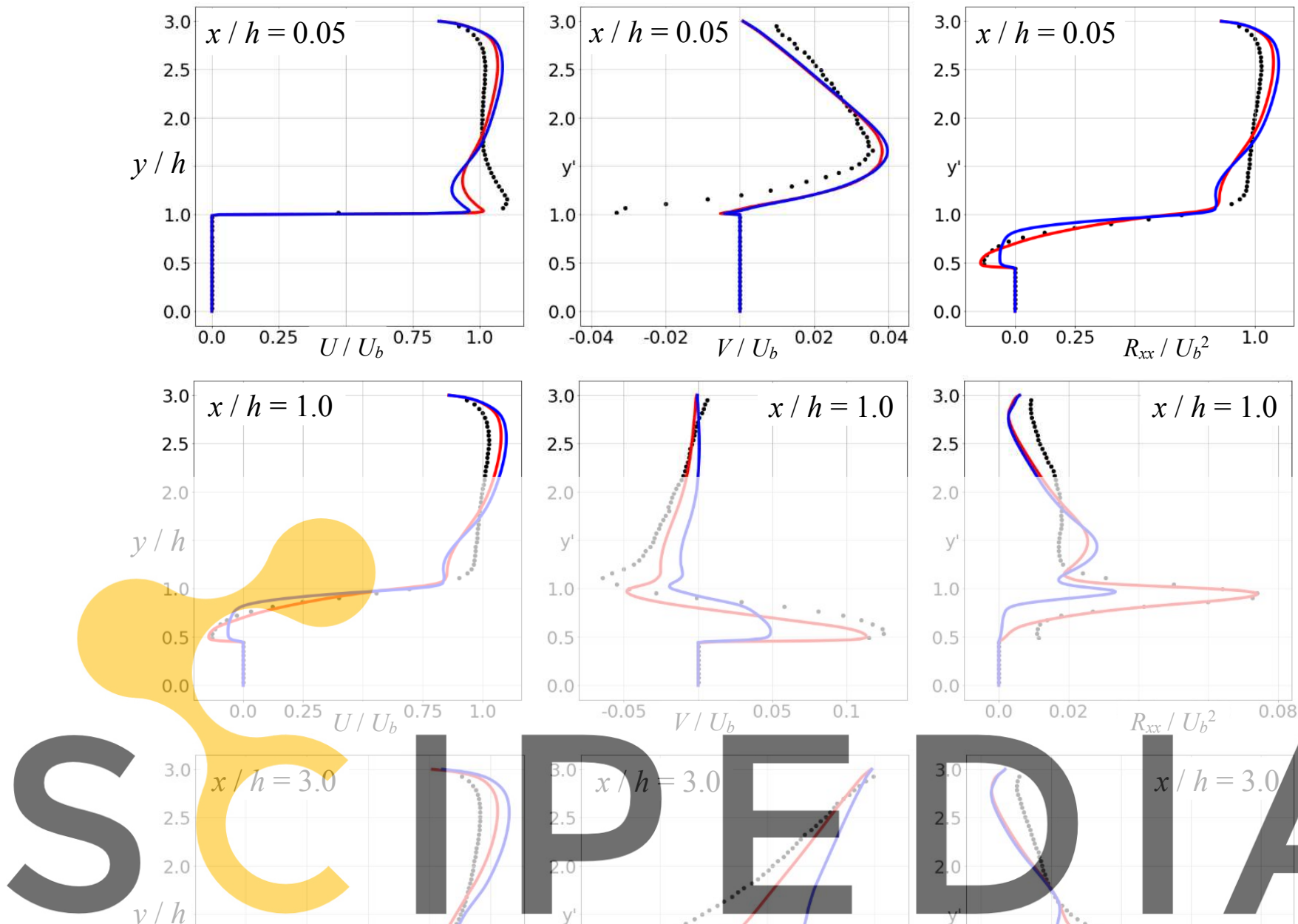

Register for free at htțps//www.scipedia.com to downtoad the version without the'watermark

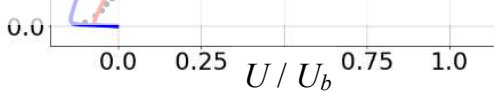

0.0

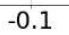

$V / U_{b}$
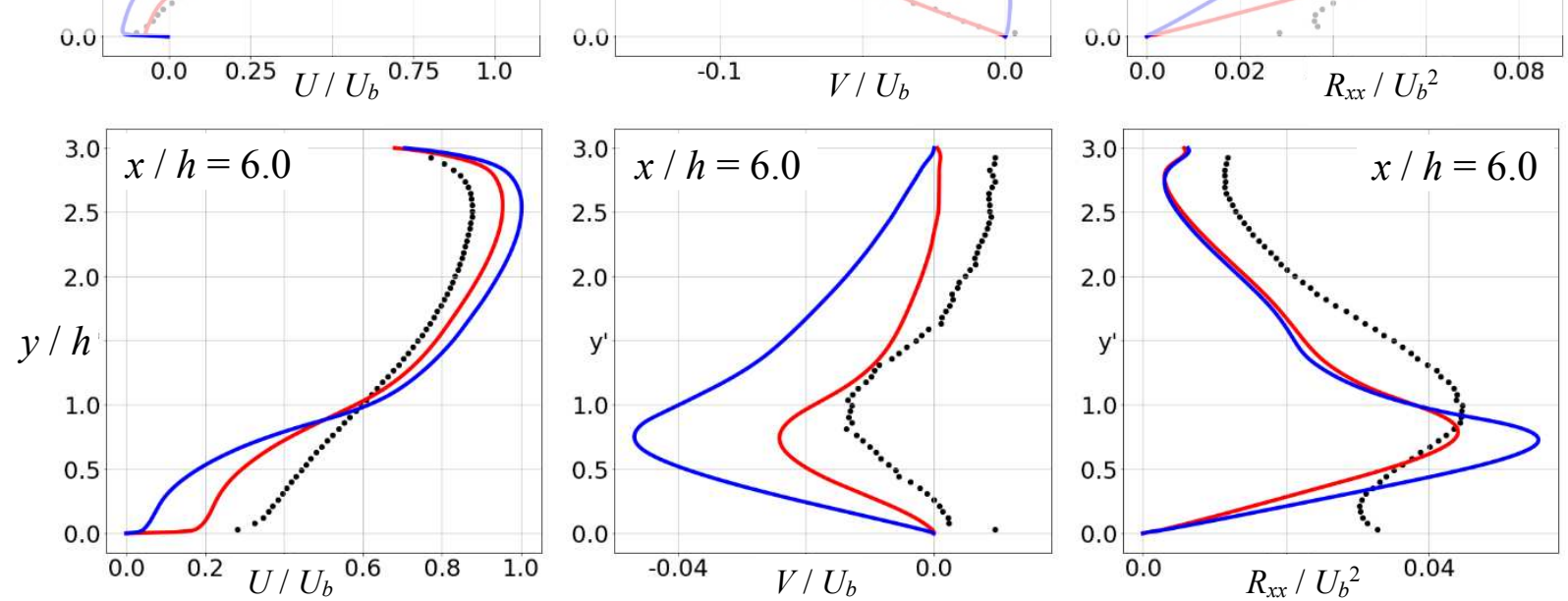

Figure 7: Streamwise velocity (left column), transverse velocity (middle column) and streamwise Reynolds stresses (right column) profiles in control sections.

Base SSG/LRR- $\omega$ model (blue), modified model (red), experiment (dots) 


\section{MODEL VALIDATION IN DIVERGING CHANNEL FLOW TEST CASE}

One of the basic tests for the modified turbulence model is subsonic separation from a smooth surface. The test case published in [8] is chosen. Present RANS computations are compared with LES data [9] and experimental data [10].

A subsonic flow past a smooth backward-facing step in a channel is studied at the Reynolds number $\operatorname{Re}=\rho_{\text {in }} U_{\text {in }} H / \mu=13600$, where $H$ is step height. In the present paper, quasi-incompressible flow was considered with Mach number $\mathrm{M}=U_{\text {in }} / c \approx 0.2$. Molecular viscosity coefficient $\mu$ was taken to be constant.

The computational mesh is based on the mesh from [9]. The distribution of nodes in the longitudinal direction was left unchanged, as well as in the transverse direction in the lower half of the channel. In the upper half of the channel, the grid nodes were obtained by reflection of the nodes from the lower half to avoid the use of wall functions. The mesh dimension is $768 \times 252=193536$ cells. Mesh overview is shown in Fig. 8. Mesh convergence study indicated that the numerical error in velocity fields lies within $1 \%$.
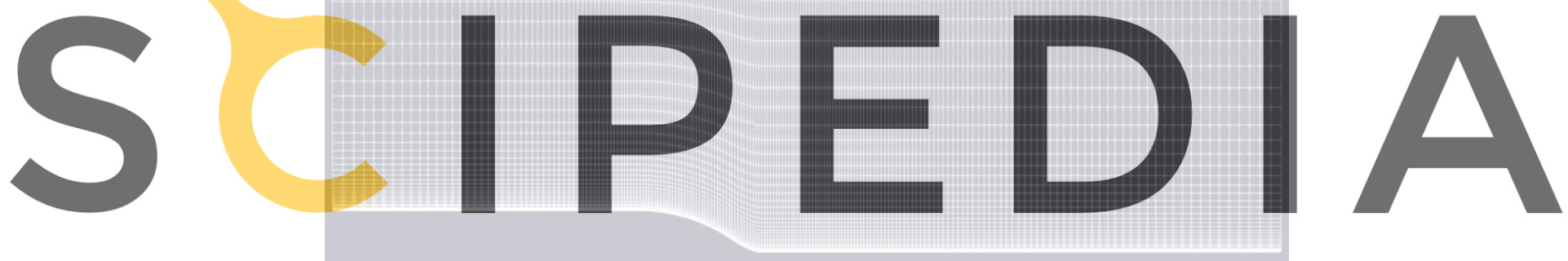

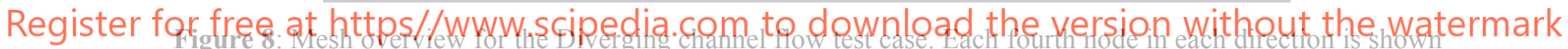

The adiabatic no-slip boundary condition was specified at the channel walls. At the outlet, constant static pressure was prescribed. At the inlet, LES profiles for velocity, pressure and temperature fields were taken. Turbulence values were obtained in preliminary plane channel flow computation with fixed velocity field from LES.

The computation using to the base model gave an overestimated length of the separation zone, the coordinate of the attachment point being $x_{r} / H=4.90$ while reference value is $x_{r} / H=4.28 \pm 0.07$. The overestimation is thus $15 \%$. In fig. 9 (top and middle), mean velocity fields and streamlines are compared obtained in LES and with the base turbulence model. The oversized separation zone is evident. In Fig. 10 (top and middle), a similar comparison of the streamwise Reynolds stress fields is given. As in the Periodic hill flow, the base model does not capture a sharp stress peak in the vicinity of separation, which leads to a slower development of the mixing layer and a larger separation compared to LES.

The transition to a modified turbulence model significantly changed the velocity and stress fields (Fig. 9 and 10, bottom). The peak value of Reynolds stress increased by $20 \%$ and 
shifted upstream from $x / H=4.92$ to $x / H=2.96$. This brought the result closer to the LES data, in which the maximum stresses were observed near $x / H=1.36$. Note that the solution could be even closer to the reference data if the separation indicator triggered earlier upstream. The change in the stress field led to the acceleration of mixing layer development and significant reduction in the separation zone length. With the new model, the reattachment point coordinate was found to be $x_{r} / H=3.80$, which is $11 \%$ lower than the reference value. An underestimation of the separation length is not typical for modern turbulence models, and this behavior opens opportunities for better adjustment of the proposed model in the future.

Finally, in Fig. 11 and 12 transverse profiles of two velocity components and streamwise Reynolds stress are plotted. As can be seen, the predictions are improved with the modified model. Only near $x / H=3$, streamwise stresses have an overshoot due to suboptimal performance of the separation indicator.

\section{CONCLUSIONS}

It is shown that within steady RANS approach, it is possible to significantly improve the subsonic separation prediction. The proposed modification of SSG/LRR- $\omega$ model does not use second velocity derivatives. Two test cases involving subsonic separation from smooth surface demonstrated that the modified model captures the kinetic energy peak near the separation zone origin missed by the original version of SSG/LRR- $\omega$. This leads to the

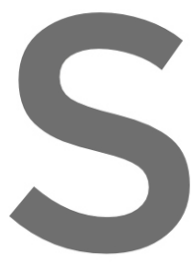
reduction of separation length prediction errors down to $11 \%$.
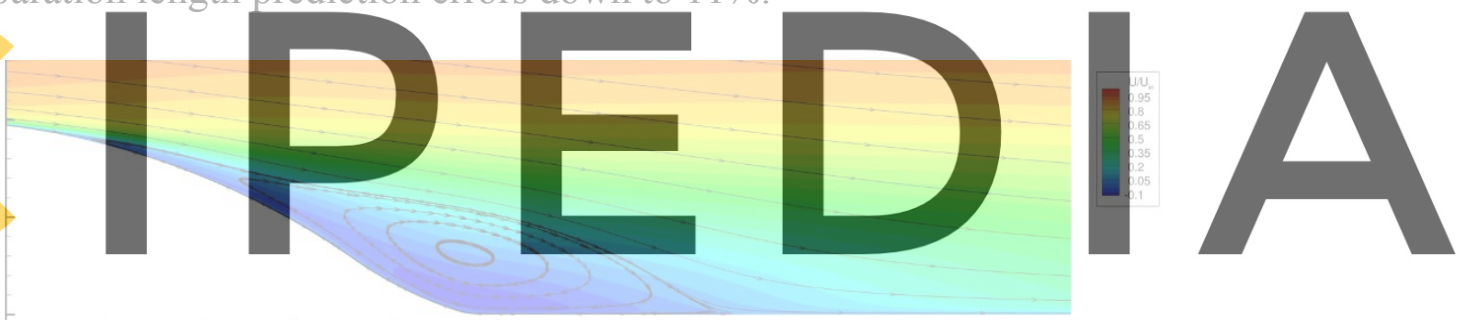

Register for free at https//www.scipedia.com to download the version without the watermark

立

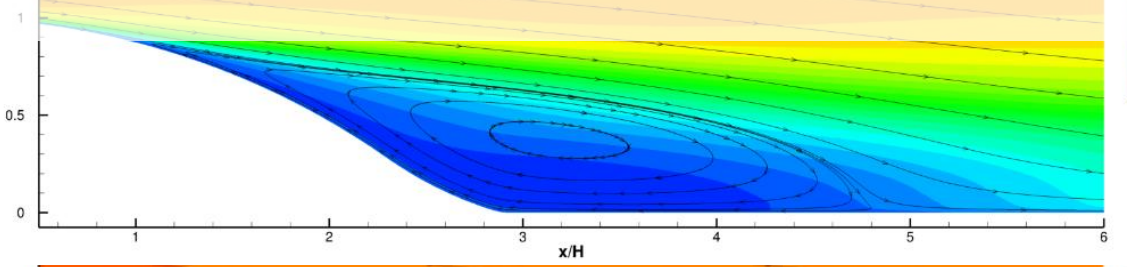

폭
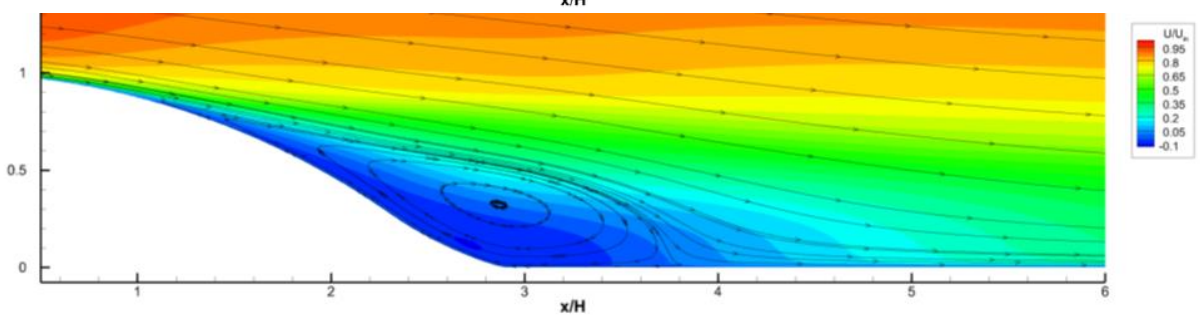

Figure 9: Mean streamwise velocity fields. LES data (top), base SSG/LRR- $\omega$ model (middle), modified model (bottom) 
$\stackrel{x}{\stackrel{x}{x}}$
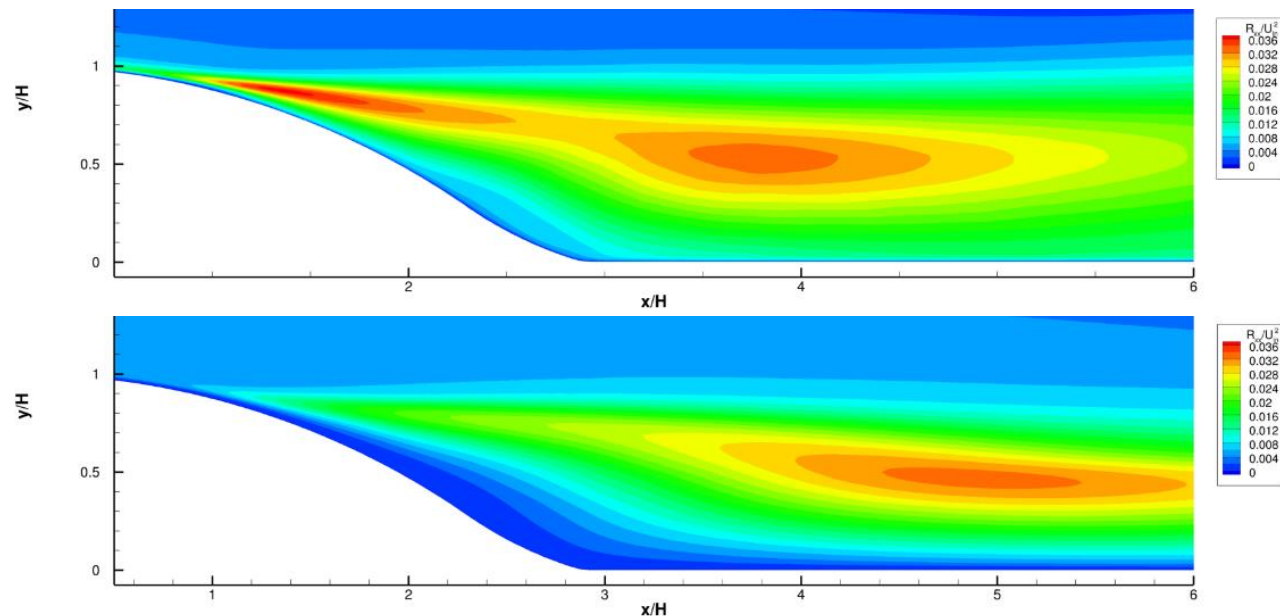

$\stackrel{x}{\frac{1}{x}}$

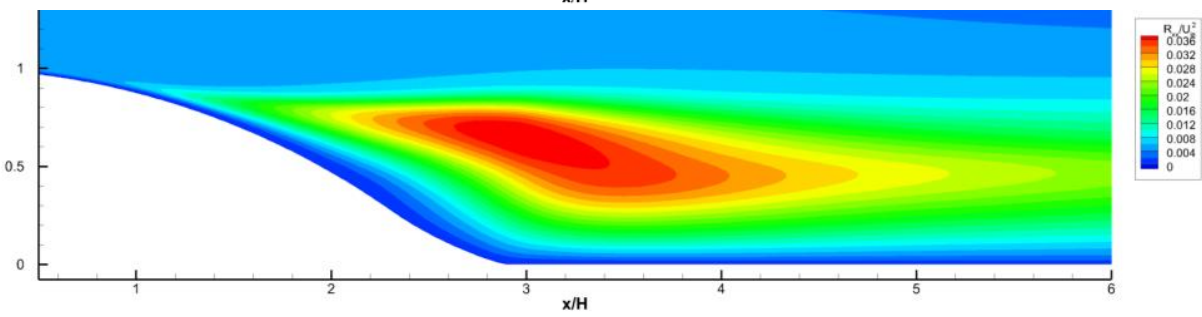

Figure 10: Streamwise Reynolds stress fields. LES data (top), base SSG/LRR- $\omega$ model (middle), modified model (bottom)
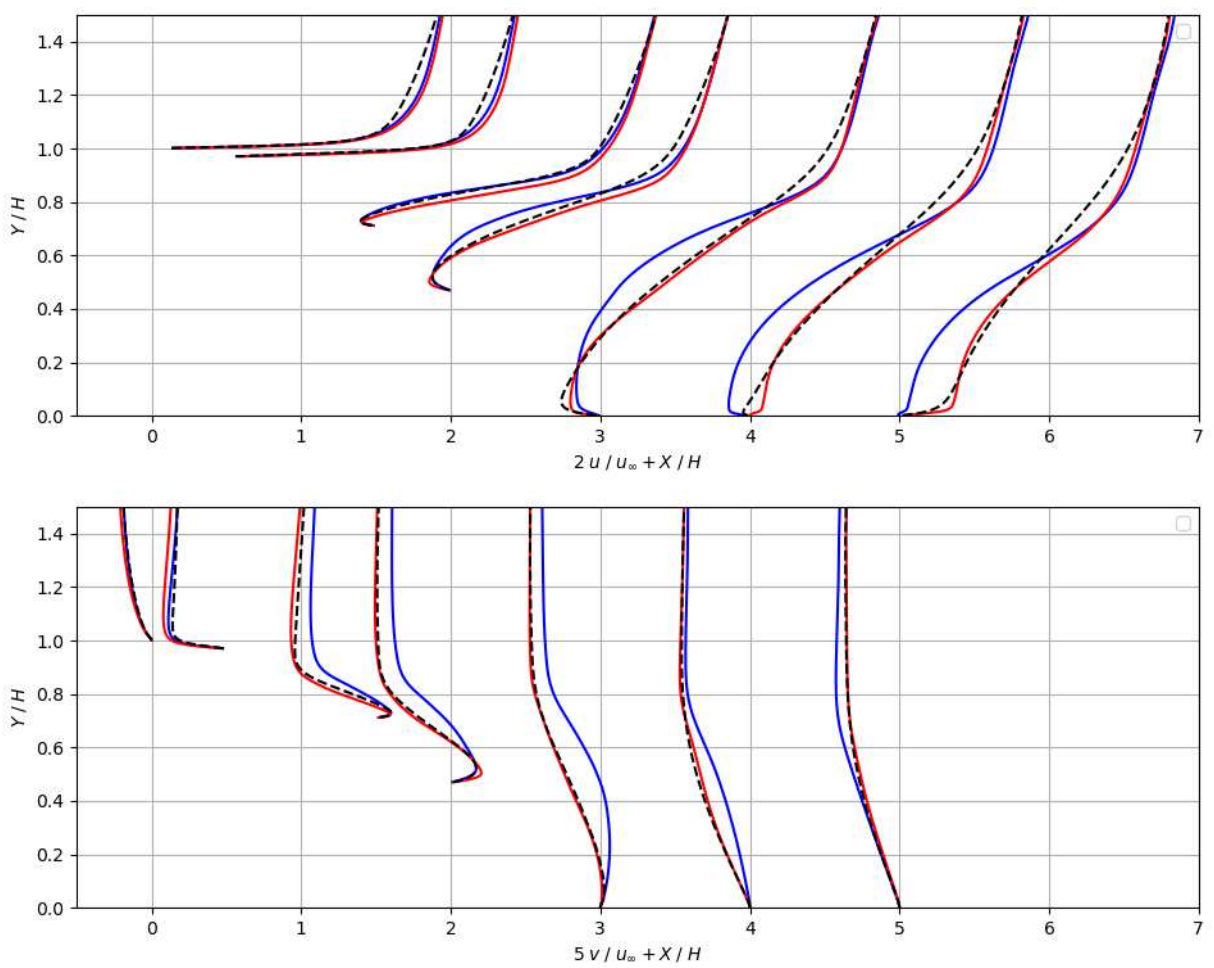

Figure 11: Streamwise (top) and transverse (bottom) velocity profiles. Base SSG/LRR- $\omega$ model (blue), modified model (red), LES data (dashed line) 


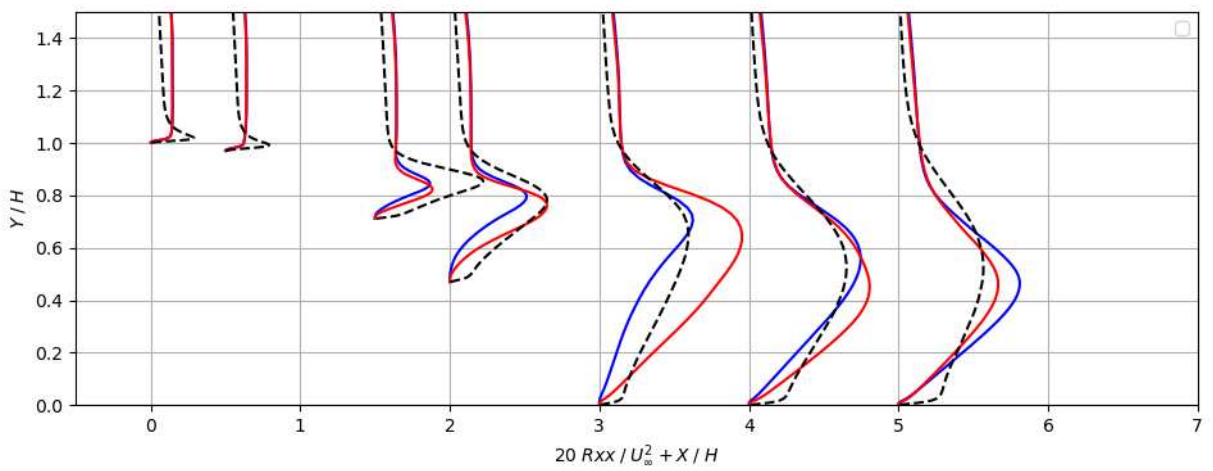

Figure 12: Streamwise Reynolds stress profiles.

SSG/LRR- $\omega$ model (blue), modified model (red), LES data (dashed line)

\section{REFERENCES}

[1] Wilcox, D.C. Turbulence modeling for CFD, $3^{\text {rd }} e d$. DCW Industries (2006).

[2] Eisfeld, B. (ed.) Differential Reynolds Stress Modeling for Separating Flows in Industrial Aerodynamics. Springer (2015).

[3] Jakirlić, S. and Maduta, R. "Steady" RANS modeling for improved prediction of wallbounded separation. AIAA J (2016) 54:1803-1809.

[4] Oster, D. and Wygnanski, I. The forced mixing layer between parallel streams. J. Fluid Mech (1982) 123:91-130.

[5] Eisfeld, B., and Rumsey, C.L. Length-scale correction for Reynolds-stress modeling. AIAA J (2020) 58:1518-1528.

[6] Menter, F.R., Kuntz, M. and Langtry, R. Ten years of industrial experience with the SST turbulence model. Turb. Heat Mass Transf (2003) 4:625-632.

[7] Breuer, M., Peller, N., Rapp, C. and Manhart, M. Flow over periodic hills-numerical and experimental study over a wide range of Reynolds numbers. Comp. Fluids (2009) 38:433-457.

[8] Bentaleb, Y., Lardeau, S. and Leschziner, M.A. Large-eddy simulation of turbulent boundary layer separation from a rounded step. J. Turb (2012) 13:1-28.

[9] https://turbmodels.larc.nasa.gov/Other_LES Data/curvedstep.html

[10] Zhang, S. and Zhong, S. An experimental investigation of turbulent flow separation control by an array of synthetic jets. AIAA Paper 2010-4582. 